\title{
Variable Structure Control with Optimized Sliding Surface for Spacecraft Slewing Maneuver
}

\author{
Sangbum Cho*, Gwanyoung Moon** and Youdan Kim*** \\ Department of Aerospace Engineering, Seoul National University \\ Seoul, Korea151-742
}

\begin{abstract}
A variable structure controller with an optimized sliding surface is proposed for slew maneuver of a rigid spacecraft. Rodrigues parameters are chosen to represent the spacecraft attitude. The quadratic type of performance index is used to design the sling surface. For optimization of the sliding surface, a Hamilton-Jacobi-Bellman equation is formulated and it is solved through the numerical algorithm using Galerkin approximation. The solution denotes a nonlinear sliding surface, on which the trajectory of the system satisfies the optimality condition approximately. Simulation result demonstrates that the proposed controller is effectively applied to the slew maneuver of a rigid spacecraft.
\end{abstract}

Key Word : Sliding mode control, Optimal sliding surface, Galerkin approximation

\section{Introduction}

The variable structure control theory has been applied successfully to the spacecraft slew maneuver problem.[1-4] The variable structure controller is composed of two control phases; the reaching phase to the sliding surface and the maintaining phase on the sliding surface. The design process of the variable structure controller is straight forward if the sliding surface is chosen. However, the variable structure control theory itself does not give guidelines to the design of the sliding surface, which is an essential factor to the performance of the controller. Therefore, the sliding surface in the variable structure control theory should be carefully selected such that it guarantees the good performance as well as the system stability.

The first introduction of the optimized sliding surface into the slew maneuver problem was carried out by Vadali.[1] In his study, the performance index was chosen as a quadratic function of the attitude vector and the angular rate vector, and a linear sliding surface was proposed as a special case under simplifying assumptions. Terui proposed the variable structure controller whose sliding surface was represented as a linear combination of the error vector.[2] MaDffie and Shtessel studied the decoupled sliding mode controller and observer using a linear sliding surface.[3] The attitude tracking problem was solved by the variable structure controller.[4] For general linear systems, it was proved that the LQR-based cost function adopted in the optimization of sliding surfaces gave the linear control law.[5]

In this paper, the slew maneuver problem is dealt with through the optimization of the sliding surface. Rodrigues parameters are used to represent the spacecraft attitude. Contrast to the existing approaches, the sliding surface is modeled as a general nonlinear function. The introduction of a general nonlinear function gives flexibility and generosity to the design of sliding surface. Hence,

* Currently Researcher, Korea Aerospace Research Institute

** Graduate student, Seoul National University

*** Professor, Seoul National University

E-mail : ydkim@snu.ac.kr, Tel : 02-880-7398, Fax : 02-887-2662 
more effective sliding surface can be designed. To solve a Hamilton-Jacobi-Bellman equation for optimization, the general nonlinear sliding surface is expanded by a series of polynomial functions, and then, the Galerkin's approximation is applied.[6] To verify the effectiveness of the proposed method, numerical simulations are performed using the data in Ref. [1].

\section{Spacecraft Dynamics and Kinematics}

Consider the rotational motion of a rigid spacecraft described by the following equations of motion:

$$
\begin{aligned}
& \dot{\omega}_{1}=\frac{I_{2}-I_{3}}{I_{1}} \omega_{2} \omega_{3}+\frac{T_{1}}{I_{1}} \\
& \dot{\omega}_{2}=\frac{I_{3}-I_{1}}{I_{2}} \omega_{3} \omega_{1}+\frac{T_{2}}{I_{2}} \\
& \dot{\omega}_{3}=\frac{I_{1}-I_{2}}{I_{3}} \omega_{1} \omega_{2}+\frac{T_{3}}{I_{3}}
\end{aligned}
$$

where $\omega_{1}, \omega_{2}$, and $\omega_{3}$ are the spacecraft angular velocities; $I_{1}, I_{2}$, and $I_{3}$ are the principal moments of inertia of the rigid spacecraft; $T_{1}, T_{2}$, and $T_{3}$ are the external control torques, respectively. The attitude kinematics are expressed in Rodrigues (or Gibbs) parameters as Eq. (4)

$$
\left[\begin{array}{l}
\dot{g}_{1} \\
\dot{g}_{2} \\
\dot{g}_{3}
\end{array}\right]=\frac{1}{2}\left[\begin{array}{ccc}
1+g_{1}^{2} & g_{1} g_{2}-g_{3} & g_{1} g_{3}+g_{2} \\
g_{2} g_{1}+g_{3} & 1+g_{2}^{2} & g_{2} g_{3}-g_{1} \\
g_{3} g_{1}-g_{2} & g_{3} g_{2}-g_{1} & 1+g_{3}^{2}
\end{array}\right]\left[\begin{array}{c}
\omega_{1} \\
\omega_{2} \\
\omega_{3}
\end{array}\right]
$$

Rodrigues parameters are chosen to represent the attitude of the spacecraft since they do not have the constraint equation as in quaternions and the expression is much simpler compared to the Euler parameters. The Rodrigues parameters can be expressed in quaternions as

$$
g=\left[\begin{array}{l}
g_{1} \\
g_{2} \\
g_{3}
\end{array}\right]=\left[\begin{array}{l}
q_{1} / q_{4} \\
g_{2} / q_{4} \\
q_{3} / q_{4}
\end{array}\right]
$$

The above equations can be rewritten in vector form for simplicity:

$$
\begin{aligned}
& \dot{\omega}=F(\omega)+u \\
& \dot{g}=G(g) \omega
\end{aligned}
$$

where $u=\left[\begin{array}{lll}u_{1} & u_{2} & u_{3}\end{array}\right]^{T}$ and $u_{i}=\frac{T_{i}}{I_{i}}$.

\section{Mathematical Formulation for Optimization}

The idea of sliding mode control is to reduce the number of variables by assuming that the trajectory moves along the sliding. The design process of a variable structure controller is done in three steps.

1. Sliding surface that can possibly meet optimality conditions is selected.

2. The controllers of the maintaining phase on the sliding surface are designed.

3. It is ensured that the state trajectory from any initial condition is forced toward the sliding surface. 
In most previous literatures on variable structure control, the sliding surfaces have been chosen in arbitrary ways or as a simple linear function. If a systematic method can be found for selecting the sliding surface, a better closed-loop performance of the controller would be obtained.

Since the rigid spacecraft model is a cascade system, the angular velocity $\omega$ can be thought of as the pseudo-control input for the attitude kinematics Eq. (4). Consider the following sliding surface defined as

$$
S=\omega-\omega^{*}(g)
$$

If states are initially on the sliding surface, the trajectory of the spacecraft attitude motion exactly follows the sliding surface; i.e.,

$$
\omega=\omega^{*}(g)
$$

The optimal problem considered in this paper can be summarized as follows.

Find a feedback control law $\omega=\omega^{*}(g)$ which minimizes the performance index

$$
J=\int_{0}^{\infty}\left[Q(g)+\omega^{T} R \omega\right] d t
$$

subject to the constraint $\dot{g}=G(g) \omega$. If an optimal control exists, it is given by

$$
\omega^{*}(g)=-\frac{1}{2} R^{-1} G^{T}(g) \frac{\partial V^{*}}{\partial g}(g)
$$

where $V$ is the solution of the Hamilton-Jacobi-Bellman (HJB) equation

$$
H J B\left(V^{*}\right)=Q-\frac{1}{4}\left(\frac{\partial V^{*}}{\partial g}\right)^{T} G R^{-1} G^{T}\left(\frac{\partial V^{*}}{\partial g}\right)=0
$$

In general, the HJB equation is difficult to solve, especially for nonlinear systems. In the following section, a numerical algorithm to solve the above HJB equation will be presented.

\section{Numerical Algorithm for Solving HJB Equation}

The numerical algorithm for solving the HJB equation is based on the Galerkin's spectral method.[6] The proposed algorithm is composed of two steps. The first step is to use successive approximation for changing the HJB equation into a sequence of linear partial differential equations. The second step is to approximate the resulting equations via Galerkin's spectral method. The following Generalized Hamilton-Jacobi-Bellman (GHJB) equation is introduced to develop the proposed algorithm.

$$
G H J B(V ; \omega)=\frac{\partial V^{T}}{\partial g} G \omega+Q+\omega^{T} R \omega=0, \quad V(0)=0
$$
equation

Note that if we plug the optimal solution $V^{*}$ and $\omega^{*}$ into the GHJB equation, it gives the HJB

$$
H J B\left(V^{*}\right)=G H J B\left(V^{*} ;-\frac{1}{2} R^{-1} G^{T} \frac{\partial V^{*}}{\partial g}\right)=0
$$

The proposed numerical algorithm requires the following initialization and iterative process described as follows: 
Initialization

Select an initial function $\omega^{(0)}(g)$ such that

- $\omega^{(0)}(g)$ is continuous

- $\omega^{(0)}(g)=0$

- The system $\dot{g}=G(g) \omega^{(0)}(g)$ is Lyapunov stable,

- The cost function $J\left(g, \omega^{(0)}\right)$ is finite for all $g$.

\section{Iterative process}

For $i \geqq 0$, find the performance index for $\omega^{(i)}$

$$
V^{(i)}\left(g ; \omega^{(i)}\right)=\int_{0}^{\infty}\left[Q(g)+\omega^{(i) T} R \omega^{(i)}\right] d \tau
$$

and update $\omega^{(i+1)}(g)$ function by solving the equation, $\operatorname{GHJB}\left(V^{(i)} ; \omega^{(i+1)}\right)=0$.

$$
\omega^{(i+1)}=-\frac{1}{2} R^{-1} G^{T} \frac{\partial V^{(i)}}{\partial g}
$$

The condition $V^{*}(g) \leqq V^{(i+1)}(g) \leqq V^{(i)}(g)$ holds for each $i \geqq 0$ and the solution does not get stuck in local minima. Therefore, if the unique optimal control $\omega^{*}(g)$ exists, $\omega^{(i)}(g)$ converges to the optimal control uniformly[6].

To apply this method numerically, the Galerkin approximation to $V^{(i)}$ is used.

$$
V_{N}^{(i)}(g)=\sum_{j=1}^{N} c_{j}^{(i)} \phi_{j}(g)
$$

where $\left\{\phi_{j}: \Omega \rightarrow R\right\}_{j=1}^{\infty}$ spans the set $\left\{f \in C^{1}: f(0)=0\right\}$. If we solve the GHJB equation, we obtain

$$
\omega_{N}^{(i+1)}(g)=-\frac{1}{2} R^{-1} G^{T}(g) \frac{\partial V_{N}^{(i)}}{\partial g}(g)
$$

It can be proven that the approximate control is guaranteed to stabilize the system if the algorithm is truncated as a finite, but large enough $i$ and $N$.

\section{Sliding Mode Control using Optimized Sliding Surface}

\section{Comparison with existing methods}

The concept of the sliding surface in the variable structure control theory is similar to that of the tracking function in the back stepping theory[7]. This similarity comes from the facts that both the sliding surface and the tracking function describe the desired relationship of state variables and the system structures are same each other. However, the sliding surface is a more restrictive and harder constraint than the tracking function. In Ref. [8], an application of the nonlinear tracking function to the back-stepping controller was demonstrated. The tracking function in Ref. [8] was designed after considering physical properties such as peak torque and settling time in time-domain. Thus, the performance of the controller has been improved through the time-response observation and the proper shaping of the tracking function. In contrast to this kind of the time-domain approach, the technique of this paper defines formally the controller performance index and then applies the optimization process.

\section{Control law design}

The optimal sliding surfaces is described by 


$$
s_{i}=\omega_{i}-\omega_{i}^{*}(g), i=1,2,3
$$

where the expression of the $\omega_{i}(g)$ is given in Eq. (21). The sliding surface vector $S$ is defined as follows:

$$
S=\left[\begin{array}{lll}
s_{1} & s_{2} & s_{3}
\end{array}\right]^{T}
$$

Time derivative of the above equation yields

$$
\dot{S}=\dot{\omega}-\left[\frac{\partial \omega^{*}}{\partial g}\right] \dot{g}=F(\omega)+u_{e q}-\left[\frac{\partial \omega^{*}}{\partial g}\right] G(g) \omega
$$

To satisfy $\dot{S}=0$ on the sliding surface, the equivalent control is given by

$$
u_{e q}=\left[\frac{\partial \omega^{*}}{\partial g}\right] G(g) \omega-F(\omega)
$$

Once the state trajectory reaches the sliding surface $S(g, \omega)=0$, the equivalent control guarantees that the trajectory remains in the sliding surface under ideal condition. Since $\omega=\omega^{*}(g)$ is a stabilizing control, the state trajectory eventually goes to zero.

The resulting variable structure control law is expressed as the sum of the equivalent control and the sign function of the sliding surface vector.

$$
u=u_{e q}+u_{s}
$$

where $u_{s}$ is expressed as

$$
u_{s}=\left[\begin{array}{l}
k_{1} \operatorname{sign}\left(s_{1}\right) \\
k_{2} \operatorname{sign}\left(s_{2}\right) \\
k_{3} \operatorname{sign}\left(s_{3}\right)
\end{array}\right], k_{i}<0
$$

However, the use of the sign function generates the chattering phenomenon. The widely-used method to reduce the chattering phenomenon is to replace the sign function by the saturation function as followings:

$$
u_{s}=\left[\begin{array}{l}
k_{1} \operatorname{sat}\left(s_{1}\right) \\
k_{2} \operatorname{sat}\left(s_{2}\right) \\
k_{3} \operatorname{sat}\left(s_{3}\right)
\end{array}\right]
$$

where the saturation function defined as

$$
\operatorname{sat}(s)=\left\{\begin{array}{llr}
1 & \text { if } & s>\epsilon \\
-1 & \text { if } & s \leftarrow \epsilon \\
s / \epsilon & \text { if }-\epsilon<s<\epsilon
\end{array}\right.
$$

\section{Numerical Simulation}

To illustrate the performance of the proposed control scheme, a numerical simulation is performed. The control objective is to stabilize the spacecraft, and it is assumed that the maximum torque about each axis is limited to $2.5 \mathrm{~N} \cdot \mathrm{m}$. The initial conditions are set as

$$
\left[\begin{array}{l}
g_{1}(0) \\
g_{2}(0) \\
g_{3}(0)
\end{array}\right]=\left[\begin{array}{l}
0.6881 \\
0.6881 \\
0.6881
\end{array}\right], \quad\left[\begin{array}{l}
\omega_{1}(0) \\
\omega_{2}(0) \\
\omega_{3}(0)
\end{array}\right]=\left[\begin{array}{l}
0.001 \mathrm{rad} / \mathrm{s} \\
0.005 \mathrm{rad} / \mathrm{s} \\
0.001 \mathrm{rad} / \mathrm{s}
\end{array}\right]
$$

The nominal values of $I_{1}, I_{2}$ and $I_{3}$ are 86,85 and $113 \mathrm{~kg} / \mathrm{m}^{2}$, respectively. To show the robustness 
of the proposed control law with respect to variations in moments of inertia, 10\%, $-5 \%$ and $15 \%$ variations of $I_{1}, I_{2}$ and $I_{3}$ are considered. The disturbance torques are assumed to be

$$
\begin{aligned}
& u_{1 D}=-0.005 \sin (t) N \cdot m \\
& u_{2 D}=0.005 \sin (t) N \cdot m \\
& u_{3 D}=-0.005 \sin (t) N \cdot m
\end{aligned}
$$

For comparison, the following conventional sliding mode controller is adopted[1]

$$
\begin{gathered}
U_{i}=-I_{i} k \omega_{i}\left|q_{0}\right| / 2-\alpha_{1 i} S_{i}-\alpha_{2 i} \operatorname{sgn}\left(S_{i}\right) \\
S_{i}=\omega_{i}+k q_{i} \quad \text { where } i=1,2,3
\end{gathered}
$$

where $k=0.25$ and the coefficients $\alpha_{11}, \alpha_{12}$, and $a_{13}$ are chosen as $10 I_{1}, 10 I_{2}$, and $2 I_{3}$, respectively, to compensate for the nonlinearities. The coefficients $a_{2 i}$ are chosen as 0.005 based on the disturbance torque magnitudes.

The threshold value $\varepsilon$ in (24) is selected as 0.001 , and the gain $k_{i}$ 's in Eq. (24) are selected as $k_{i}=-2$. To design the optimal sliding surfaces, we choose a quadratic performance index as

$$
J=\frac{1}{2} \int_{0}^{\infty}\left(g_{1}^{2}+g_{2}^{2}+g_{3}^{2}+\omega_{1}^{2}+\omega_{2}^{2}+\omega_{3}^{2}\right) d t
$$

To apply the numerical algorithm for solving the HJB equations, even symmetric polynomials up to 4th degree are taken as basis functions.

$$
\begin{gathered}
\{\phi\}=\left\{g_{1:}^{2} g_{2}^{2}, g_{3}^{2}, g_{1} g_{2}, g_{2} g_{3}, g_{3} g_{1}, g_{1}^{4}, g_{2}^{4}, g_{3}^{4}, g_{1}^{3} g_{3}, g_{2}^{3} g_{1}, g_{2}^{3} g_{3}, g_{3}^{3} g_{1}, g_{3}^{3} g_{2},\right. \\
\left.g_{1}^{2} g_{2}^{2}, g_{2}^{2} g_{3}^{2} g_{3}^{2} g_{1}^{2} g_{1}^{2} g_{2} g_{3}, g_{2}^{2} g_{1} g_{3,} g_{3}^{2} g_{1} g_{2}\right\}
\end{gathered}
$$

The initial sliding surface is assumed as

$$
\omega^{(0)}=\left[\begin{array}{l}
-g_{1} \\
-g_{2} \\
-g_{3}
\end{array}\right]
$$

After a few iterations, we obtain the following optimal solution.

$$
\omega^{*}(g)=\left[\begin{array}{c}
0.0514 g_{1}^{5}+\left(-0.1191+0.0781 g_{2}^{2}+0.0781 g_{3}^{2}\right) g_{1}^{3}+\left(0.0514 g_{2}^{4}-0.1315 g_{3}^{2}-0.1705\right. \\
\left.-0.1315 g_{2}^{2}+0.0514 g_{3}^{4}+0.0781 g_{2}^{2} g_{3}^{2}\right) g_{1}-0.0124 g_{2} g_{3}^{3}+0.0124 g_{2}^{3} g_{3} \\
0.0514 g_{1}^{4} g_{2}-0.0124 g_{1}^{3} g_{3}+\left(-0.1315 g_{2}+0.0781 g_{2}^{3}+0.0781 g_{3}^{2} g_{2}\right) g_{1}^{2}+0.0124 g_{1} g_{3}^{3} \\
-0.1705 g_{2}-0.1191 g_{2}^{3}-0.1315 g_{3}^{2} g_{2}+0.0514 g_{2}^{5}+0.0514 g_{3}^{4} g_{2}+0.0781 g_{3}^{2} g_{2}^{3} \\
0.0514 g_{1}^{4} g_{3}+0.0124 g_{1}^{3} g_{2}+\left(-0.1315 g_{3}+0.0781 g_{2}^{2} g_{3}+0.0781 g_{3}^{3}\right) g_{1}^{2}-0.0124 g_{1} g_{2}^{3} \\
+0.0514 g_{3}^{5}-0.1315 g_{2}^{2} g_{3}-0.1705 g_{3}-0.1191 g_{3}^{3}+0.0514 g_{2}^{4} g_{3}+0.0781 g_{3}^{3} g_{2}^{2}
\end{array}\right]
$$

Figure 1 shows the trajectory of the value of Rodrigues parameters. The required final condition of the slew maneuver is achieved asymptotically. Figure 2 shows the time history of angular velocity vector. The abrupt changes at about ten seconds are caused by the switching form the reaching phase to the maintaining phase. Figures 3 and 4 shows $g_{1}-\omega_{1}$ plots of the conventional sliding mode controller and proposed sliding mode control law, respectively, Figures 3 and 4 clearly show the switching effect of each control law. Figures 5 and 6 show the control input histories of each control law. There are jumps at the switching time, and then the control torques converge to zero. Figure 5, the result of the conventional sliding mode controller[1], shows that the control input is saturate. However, as shown in Fig. 6, the proposed sliding mode controller regulates the states without exceeding the control saturation limit. By optimizing the sliding surface, the less control torques are required, and the smaller settling time is achieved. 


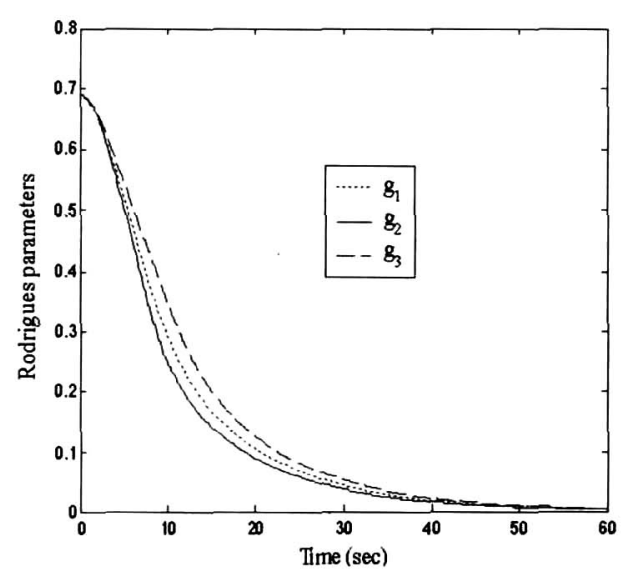

Fig. 1. Trajectory of Rodrigues Parameters

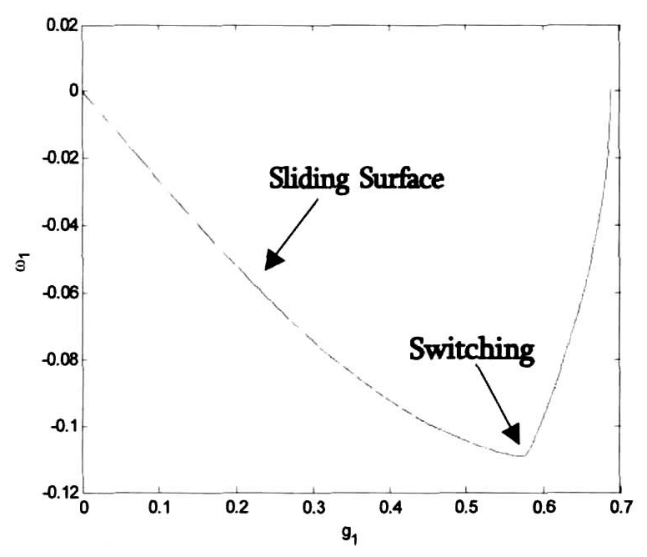

Fig. 3. State trajectory in the $g_{1}-\omega_{1}$ plane (Conventional VSC)

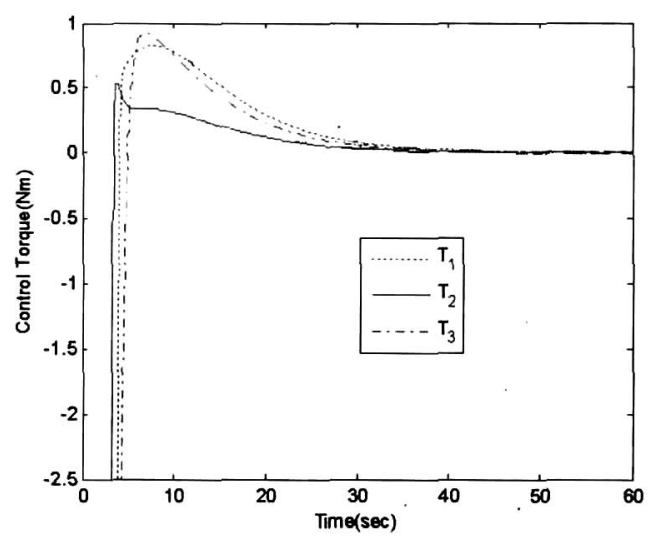

Fig. 5. Time History of Control Torque Vector (Conventional VSC)

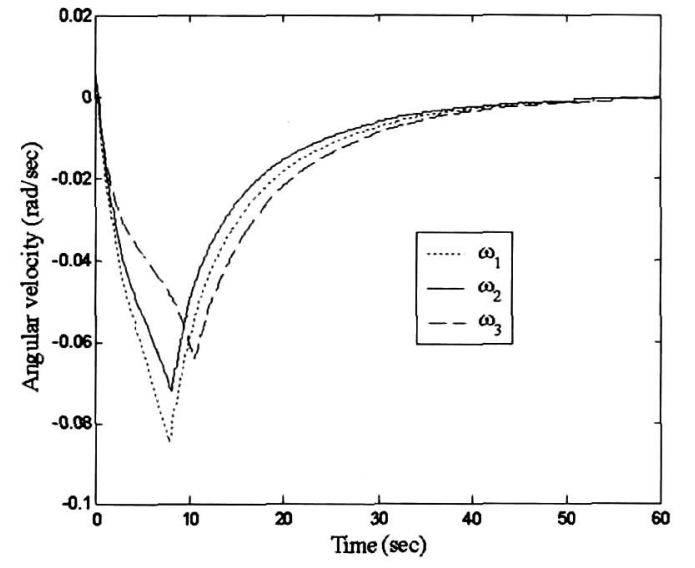

Fig. 2. Time History of Angular Velocity Vector

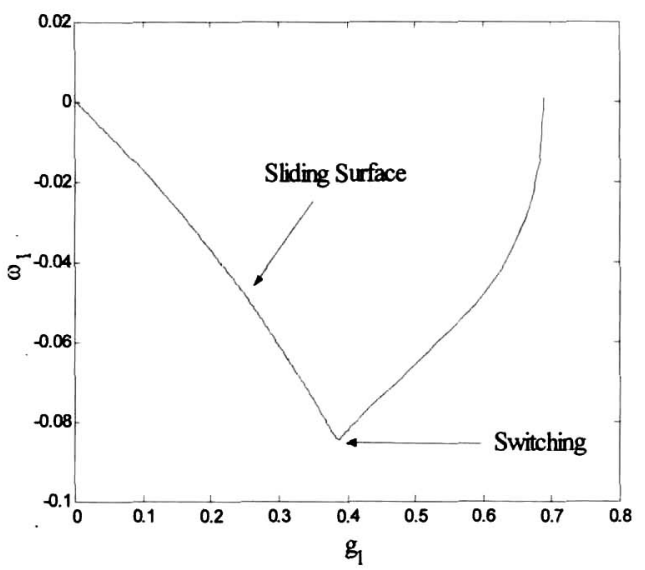

Fig. 4. State trajectory in the $g_{1}-\omega_{1}$ plane (Optimum VSC)

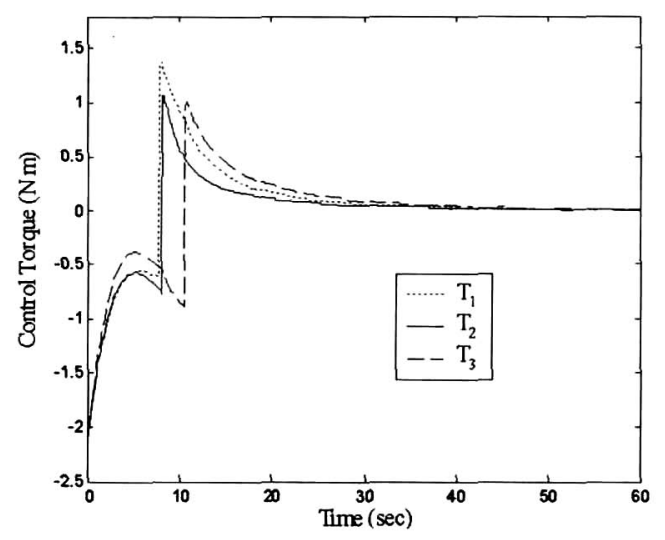

Fig. 6. Time History of Control Torque Vector (Optimum VSC) 


\section{Conclusion}

A slew maneuver controller based on the variable structure controller is proposed. To obtain an effective sliding surface, the optimal problem and the Hamilton-Jacobi-Bellman equation are investigated. The Hamilton-Jacobi-Bellman equation is solved through the numerical method using Galerkin's spectral approximation. The polynomial functions are selected as basis functions and it is shown the numerical solution of the HJB equation converges to the true solution under large indices. The concept of the proposed method is compared with that of existing methods. Numerical simulation results demonstrate that the proposed variable structure controller effectively achieve the slew maneuver of a rigid spacecraft.

\section{Acknowledgement}

This work was supported by Grant No. R01-2006-000-10189-0 from the Basic Research Program of the Korea Science \& Engineering Foundation.

\section{References}

1. S. R., Vadali, "Variable-Structure Control of Spacecraft Large-Angle Maneuvers", Journal of Guidance, Control, and Dynamics, Vol. 9, No. 2, 1986, pp. 235-239.

2. F. Terui, "Position and Attitude Control of a Spacecraft by Sliding Mode Control", Proceedings of the American Control Conference, Philadelphia, PA, 1998, pp. 217-221.

3. J. H. McDuffie, and Y. B. Shtessel, "A De-coupled Sliding Mode Controller and Observer for Satellite Attitude Control", Proceedings of the 29th Southeastern Symposium on System Theory, Cookeville, TN, 1997, pp. 92-97.

4. Y. Chen, and S. Lo, "Sliding-Mode Controller Design for Spacecraft Attitude Tracking Maneuvers", IEEE Transactions on Aerospace and Electronic Systems, Vol. 29, No. 4, 1993, pp. $1328-1333$.

5. P. Korondi, and H. Hashimoto, "Optimal Sliding Mode Design for Motion Control", IEEE Transaction on Control Systems Technology, Vol. 5, No. 2, 1996, pp. 277-282.

6. Beard, R., Saridis, G., Wen, J., "Approximate Solutions to the Time-Invariant Hamilton -Jacobi-Bellman Equations", Journal of Optimization Theory and Application, Vol. 96, No. 3, 1998, pp. 589-626.

7. Khalil, H. K., Nonlinear Systems, 2nd edition, Prentice Hall, Upper Saddle River, NJ, 1996, Chapter 13.

8. K. Kim, and Y. Kim, "Backstepping Control of Rigid Spacecraft Slew Maneuver", AIAA Guidance, Navigation, and Control Conference, Montreal, Canada, 2001. 